\title{
Estimación de parámetros eléctricos transitorios de un transformador utilizando ajuste de curvas con optimización no lineal
}

Fecha de recepción: 26-02-2021 • Fecha de aceptación: 22-04-2021 • Fecha de publicación: 10-06-2021

\author{
Víctor Hugo Tibanlombo Timbila ${ }^{1}$ \\ Escuela Politécnica Nacional, Ecuador \\ victor.tibanlombo@epn.edu.ec \\ https://orcid.org/0000-0001-9463-7587
}

Andrés Alfredo Guevara Betancourt ${ }^{2}$ Escuela Politécnica Nacional, Ecuador andres.guevara@epn.edu.ec https://orcid.org/0000-0002-2657-6441

Juan David Ramírez Guasgua ${ }^{3}$ Escuela Politécnica Nacional, Ecuador juan.ramirezd@epn.edu.ec https://orcid.org/0000-0002-9855-9835

\section{RESUMEN}

Este estudio plantea una alternativa de consecución de los parámetros eléctricos en modelos de transformadores trifásicos mediante registros obtenidos con lecturas de aparatos de medición en sistemas de protección y monitoreo asociados al transformador eléctrico. El posible uso de este procedimiento se focaliza en la estimación del modelo eléctrico en transformadores trifásicos durante su operación y funcionamiento en un sistema eléctrico y su respuesta frente a eventos transitorios. Esta propuesta permitirá aminorar recursos y uso de tiempo en comparación con metodologías existentes; puesto que, éstas usualmente necesitan que el transformador este fuera de servicio para la conexión 
de equipo especializado de alto costo, siendo muchas veces necesario trasladar el transformador a un laboratorio especializado.

Los trabajos existentes proporcionan modelos completos del transformador para simulación de eventos transitorios, estos se usaron como datos de partida para sintonizar los parámetros en MATLAB-SIMULINK. Evaluada la efectividad y confiabilidad del estudio, éste será de gran ayuda en la determinación de parámetros eléctricos transitorios en transformadores nuevos o con un tiempo considerable de funcionamiento, pues debido al uso del equipo y las condiciones de operación, los parámetros eléctricos pueden variar con respecto a sus registros de fábrica.

KEYWORDS: transformador, alta frecuencia, función de transferencia, parámetros eléctricos transitorios

\section{ABSTRACT}

This study proposes an alternative to obtain the electrical parameters in three-phase transformer models by means of records obtained with readings from measuring devices in protection and monitoring systems associated with the electrical transformer. The possible use of this procedure focuses on the estimation of the electrical model in three-phase transformers during their operation and functioning in an electrical system and their response to transient events. This proposal will reduce resources and time use in comparison with existing methodologies, since these usually require the transformer to be out of service for the connection of high-cost specialized equipment, being often necessary to move the transformer to a specialized laboratory.

Existing works provide complete transformer models for simulation of transient events, these were used as starting data to tune the parameters in MATLAB-SIMULINK. Evaluated the effectiveness and reliability of the study, it will be of great help in the determination of transient electrical parameters in new transformers or with a considerable time of operation, because due to the use of the equipment and the operating conditions, the electrical parameters may vary with respect to their factory records.

\section{PALABRAS CLAVE: transformer, high voltage, transfer function, electric transient parameters}




\section{Introducción}

Los trasformadores eléctricos han ejercido un papel fundamental en la evolución de los sistemas eléctricos de potencia y distribución. Al operar ininterrumpidamente, estos están expuestos a diferentes eventos o anomalías que pueden afectar su desempeño y vida útil, dentro de estos eventos se pueden nombrar a los eventos transitorios.

Los que se presentan en los sistemas eléctricos se encuentran en el rango de $0.1 \mathrm{~Hz}$ hasta alrededor de $50 \mathrm{MHz}$, es decir, hay eventos de baja, media y alta frecuencia (Galván, 2012), las ondas de alta frecuencia generalmente se generan por la incidencia de eventos atmosféricos en algún punto del sistema, y son transferidas a través de las líneas de transporte de energía, pudiendo alcanzar a equipos vitales en la transmisión y distribución de energía eléctrica como los transformadores (Abed, N.Y et al, 2010).

Por ello, es importante conocer el comportamiento de los transformadores mediante estudios y simulaciones de sobrevoltajes en redes eléctricas, por lo que la representación o modelación es un hito importante para la finalidad de estos estudios. Generalmente los parámetros en los modelos transitorios no son provistos en la documentación general de los equipos, y para su obtención se necesita realizar una serie de pruebas con equipos y elementos de medida especializados, que, según su precisión, proporcionarán unos valores con errores máximos permitidos. También, se debe considerar el alto costo generado por realizar estas pruebas en laboratorios especializados, pues los equipos de medida pueden variar sus lecturas de acuerdo a las condiciones climáticas y geográficas de la instalación (NMX-J-169-ANCE, 2004).

Como solución a esta problemática, se propone evaluar el performance de un transformador frente a un evento transitorio, simulando una descarga atmosférica en un laboratorio de alto voltaje. El evento se encuentra en el rango de las altas frecuencias desde los $10 \mathrm{kHz}$ hasta los $3 \mathrm{MHz}$ (Galván, 2012). Se busca obtener los datos medidos de entrada y salida en el transformador bajo prueba y posteriormente determinar los parámetros eléctricos transitorios mediante la modelación del transformador en MATLAB-SIMULINK y el uso de herramientas para ajuste de curvas con optimización no lineal.

\section{Metodología}

A partir de esta sección se detalla el proceso desarrollado para conseguir el objetivo de este artículo que es el de encontrar los parámetros eléctricos transitorios de un transformador.

\subsection{Modelo de transformador de alta frecuencia utilizado}

Para este estudio se utilizó un modelo Black-Box (Mork et al., 2007) para calcular los parámetros del transformador, para ello se ingresa la señal por el lado de alto voltaje obteniendo una curva de respuesta en el lado secundario o de bajo voltaje. Con el software MATLAB-SIMULINK y la herramienta System Identification Toolbox podemos resolver las ecuaciones que representan al modelo, esto mediante las curvas antes mencionadas, pudiendo así sintonizar los parámetros 
eléctricos.

El modelo Black-Box del transformador en alta frecuencia Two-Port de Biernacki y Czarkowski (2001) se utilizó en el presente estudio, pues este modelo es utilizado en varios estudios publicados en la IEEE, además de ser sencillo de entender, como se puede observar en la Figura 1, este modelo nos sirve en la metodología presentada, pues se pueden representar los parámetros eléctricos de un transformador trifásico en su modelo T por fase equivalente.

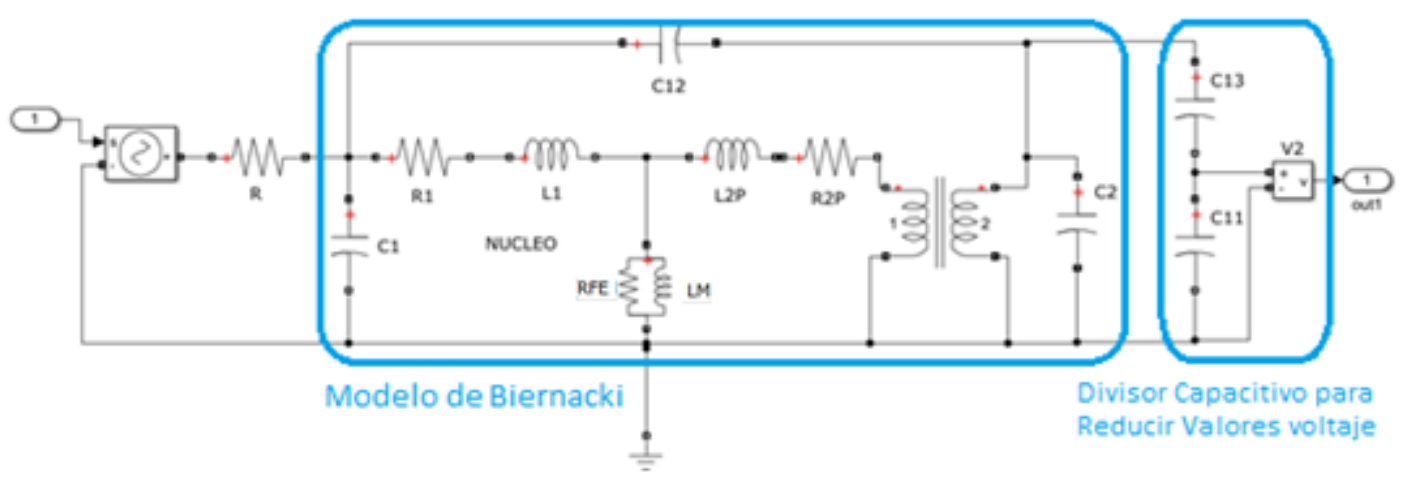

Figura 1. Modelo matemático de transformador utilizado para la simulación en MATLAB-SIMULINK.

Fuente: Matlab-Simulink

De la Figura 1:

$C_{1} \quad$ Capacitor entre devanado primario y tierra.

$C_{2} \quad$ Capacitor entre devanado secundario a tierra.

$C_{12}$ Capacitor entre devanados.

$C_{13} C_{11}$ Divisor capacitivo

$L_{1}, L_{2}$ Inductancia del devanado primario y secundario.

$R_{1}, R_{2}$ Resistencia del devanado primario y secundario.

$L_{M} \quad$ Inductancia de magnetización del núcleo.

$R_{F E} \quad$ Resistencia equivalente de pérdidas en el núcleo.

Las capacitancias $\mathrm{C} 1, \mathrm{C} 2$ y $\mathrm{C} 12$ se presentan cuando en el transformador circulan magnitudes de voltaje y corriente con alta frecuencia; las capacitancias $\mathrm{C} 13$ y $\mathrm{C} 11$ representan un divisor capacitivo acoplado para medir la curva de salida o respuesta en el lado de bajo voltaje del transformador y poder visualizar la misma en un osciloscopio digital.

\subsection{Simulación de descarga atmosférica}

En la Figura 2 se observan los elementos y su distribución para la generación de impulsos estándar que representen una descarga atmosférica en el laboratorio de alto voltaje. 


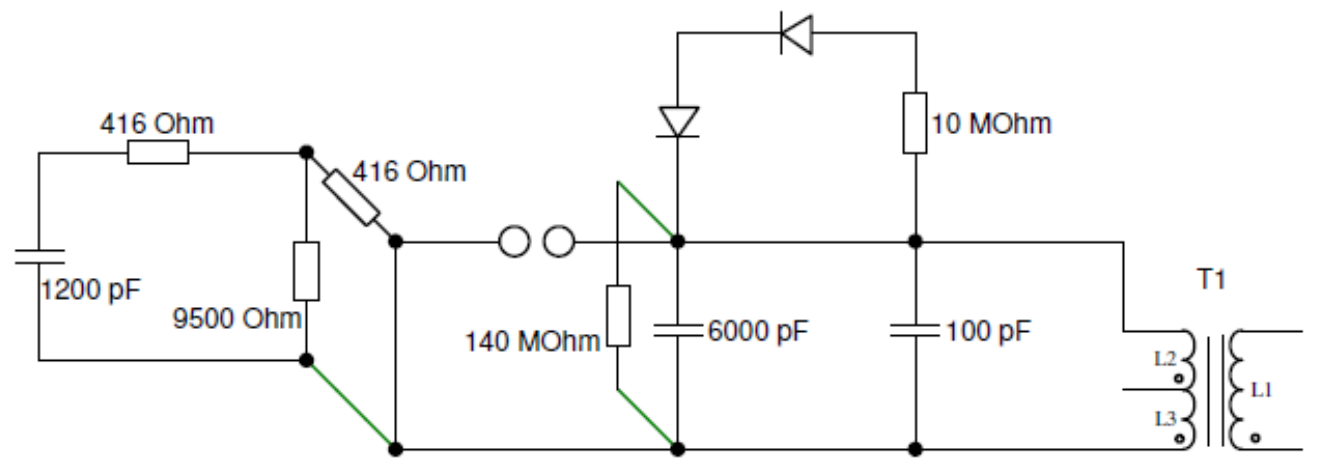

Figura 2. Arreglo de generador de impulsos de alto voltaje

Fuente: Tibanlombo (2018)

De acuerdo a la Figura 2, se utilizó un generador de impulsos que produce ondas de voltaje de frente rápido normalizadas según la IEC 60060-1, el circuito genera ondas como la de la Figura 4. Esta señal de impulso ingresa por el lado de alto voltaje al transformador y su respuesta se obtiene en su lado secundario, ambas señales son medidas mediante el canal del osciloscopio, esto se puede ver en la Figura 3.

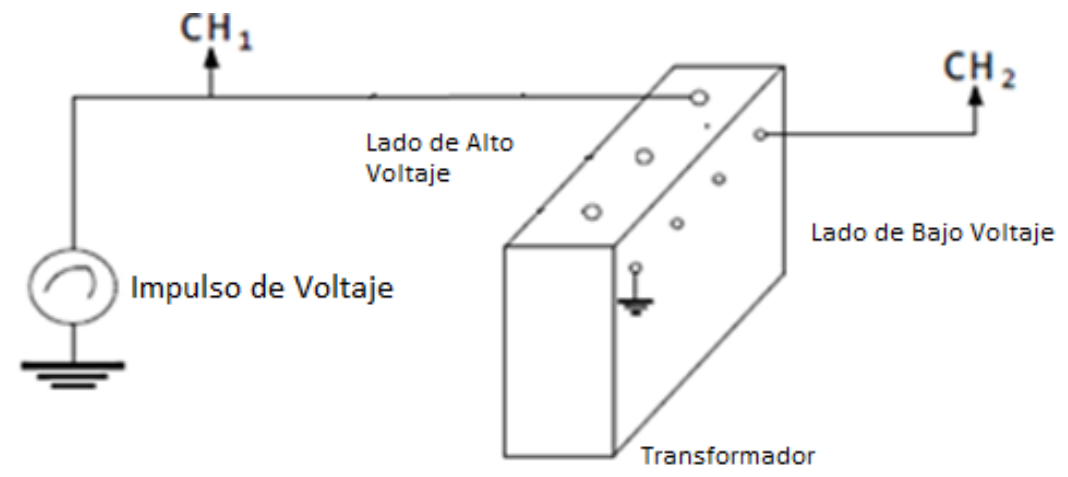

Figura 3. Arreglo de conexión de los canales del osciloscopio

Fuente: elaboración propia 


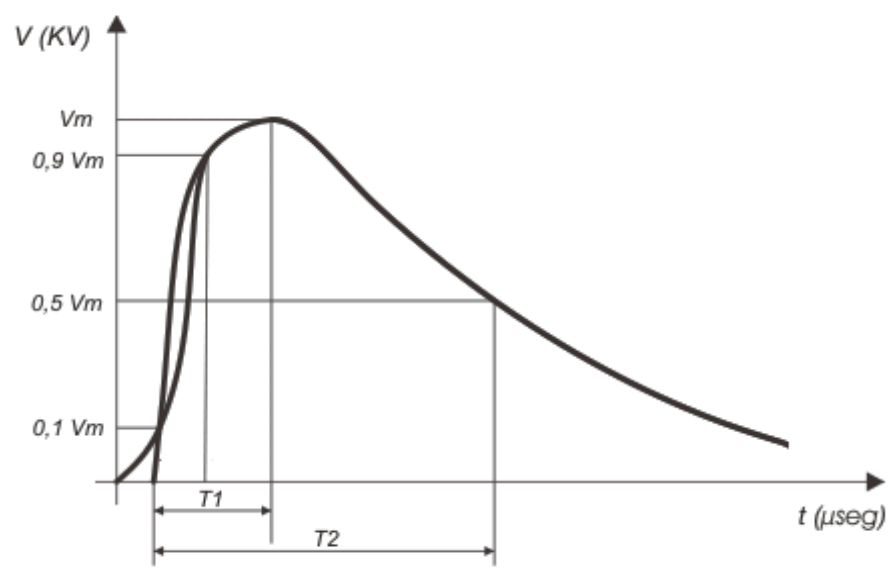

Figura 4. Forma de onda del impulso generado según IEC60-1.

Fuente: elaboración propia.

\subsection{Estimación de parámetros forma tradicional}

Para iniciar la estimación se requieren los parámetros base o de partida. Los parámetros de la Tabla 1 fueron calculados a partir de las pruebas de cortocircuito y circuito abierto practicadas al transformador de $50 \mathrm{kVA}$ acorde a la norma IEEE Std C57.12.90.

Tabla 1.

Parámetros Calculados

\begin{tabular}{|l|l|}
\hline R1 & $8.87 \Omega$ \\
\hline R2P & $8.87 \Omega$ \\
\hline R2 & $13.11 \mathrm{~m} \Omega$ \\
\hline RFE & $360 \mathrm{k} \Omega$ \\
\hline L1 & $34.06 \mathrm{mH}$ \\
\hline L2P & $34.06 \mathrm{mH}$ \\
\hline L2 & $50.38 \mathrm{uH}$ \\
\hline LM & $90.21 \mathrm{H}$ \\
\hline $\begin{array}{l}\text { R2P, } \\
\text { L2P son } \\
\text { parámetros } \\
\text { referidos } \\
\text { al lado } \\
\text { primario }\end{array}$ & \\
\hline
\end{tabular}

Fuente: elaboración propia

Además de los datos obtenidos mediante pruebas estándar, se tiene de referencia la placa del transformador de $50 \mathrm{kVA}$ que muestra los valores de fábrica del mismo, esto se observa en la Tabla 2. 
Tabla 2.

Datos de Placa

\begin{tabular}{|c|c|c|c|c|c|c|c|c|}
\hline \multicolumn{8}{|c|}{ TRANSFORMADOR TRIFÁSICO } & \\
\hline \multicolumn{3}{|c|}{ Marca } & \multicolumn{2}{|c|}{ ELIN } & \multicolumn{2}{|l|}{ Año } & \multicolumn{2}{|l|}{1965} \\
\hline \multicolumn{3}{|c|}{ Tiро } & \multicolumn{2}{|c|}{ OD $51 / 10$} & \multicolumn{2}{|l|}{$\mathrm{N}^{\circ}$ serie } & \multicolumn{2}{|c|}{1077117} \\
\hline \multicolumn{3}{|c|}{ Frecuencia } & 60 & $\mathrm{~Hz}$ & \multicolumn{2}{|l|}{ Conexión } & \multicolumn{2}{|c|}{ DY5 } \\
\hline \multicolumn{3}{|c|}{ Potencia } & 50 & kVA & \multicolumn{2}{|l|}{ Impedancia } & 3.95 & $\%$ \\
\hline \multicolumn{5}{|c|}{ Primario } & \multicolumn{4}{|l|}{ Secundario } \\
\hline 1 & 6300 & & \multirow{5}{*}{4.82} & \multirow{5}{*}{ A } & \multirow{5}{*}{231.133} & \multirow{5}{*}{ V } & \multirow{5}{*}{125.2} & \multirow{5}{*}{ A } \\
\hline 2 & 6150 & & & & & & & \\
\hline \multicolumn{2}{|l|}{3} & $\mathrm{~V}$ & & & & & & \\
\hline \multicolumn{2}{|c|}{6000} & & & & & & & \\
\hline 4 & 5700 & & & & & & & \\
\hline
\end{tabular}

Fuente: Tibanlombo (2018)

\subsection{Estimación de capacitancias}

Para estimar las capacitancias iniciales se les calculó en base al modelo de Moziful para bajas frecuencias expuesto en la Figura 5 y con su función de transferencia correspondiente empleando la ecuación (1) y también el modelo para medias frecuencias de la Figura 6 y su función de transferencia que se describe en la ecuación (2), las cuales se exponen a continuación (Moziful et al., 1997):

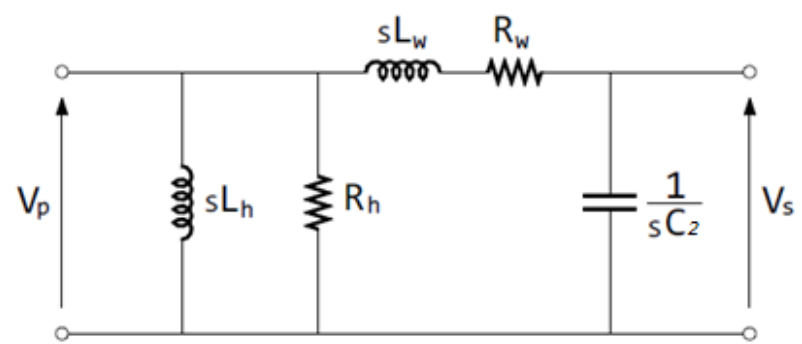

Figura 5. Modelo en baja frecuencia equivalente de Moziful.

Fuente: Moziful et al (1997) 


$$
\begin{gathered}
\frac{V_{s}}{V_{p}}= \\
\frac{s \frac{1}{C_{2} L_{w}}}{s^{3}+s^{2}\left(\frac{R_{w}}{L_{w}}+\frac{1}{C_{2} R_{h}{ }^{\prime}}\right)+s\left(\frac{1}{C_{2} L_{w}}+\frac{R_{w}}{C_{2} R_{h}{ }^{\prime} L_{w}}+\frac{1}{L_{h}{ }^{\prime} C_{2}}\right)+\left(\frac{R_{w}}{C_{2} L_{w} L_{h}{ }^{\prime}}\right)}
\end{gathered}
$$

Función de Transferencia del modelo en media frecuencia de Moziful

Donde:

$V_{S} \quad$ Voltaje de secundario o de bajo voltaje.

$V_{p} \quad$ Voltaje de primario o de alto voltaje.

$C_{2} \quad$ Capacitor entre devanado secundario a tierra.

$R_{w} \quad$ Resistencia del devanado.

$L_{w} \quad$ Inductancia del devanado.

$R_{h} \quad$ Resistencia del núcleo.

$L_{h} \quad$ Inductancia del núcleo.

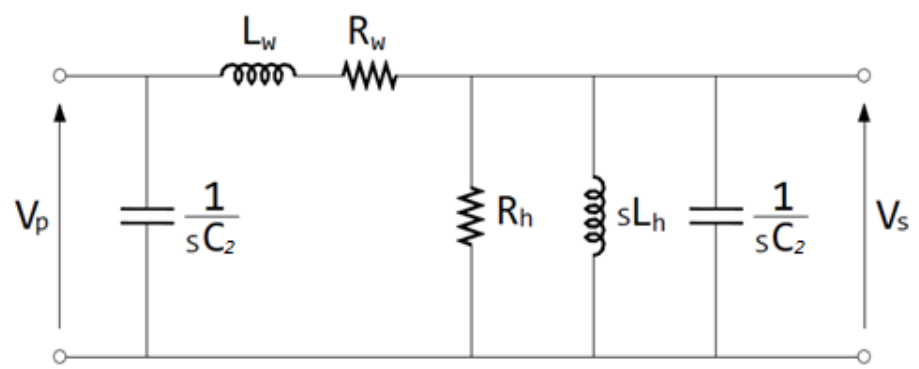

Figura 6. Modelo de Moziful equivalente para el transformador de media frecuencia.

Fuente: Moziful et al (1997)

$$
\frac{V_{s}}{V_{p}}=\frac{\frac{1}{C_{2} L_{w}}}{s^{2}+s \frac{R_{w}}{L_{w}}+\frac{1}{C_{2} L_{w}}}
$$

Función de Transferencia en baja frecuencia del modelo de Moziful.

\section{Donde:}

$V_{S} \quad$ Voltaje de secundario o de bajo voltaje.

$V_{p} \quad$ Voltaje de primario o de alto voltaje.

$C_{2} \quad$ Capacitor entre devanado secundario a tierra.

$R_{w} \quad$ Resistencia del devanado.

$L_{w} \quad$ Inductancia del devanado.

$R_{h} \quad$ Resistencia del núcleo.

$L_{h} \quad$ Inductancia del núcleo.

Con la herramienta System Identification Toolbox (Ident) de MATLAB-SIMULINK se procedió a resolver las funciones de transferencia y así poder obtener los valores de las capacitancias iniciales de la Tabla 3. 
Tabla 3.

Capacitancias Estimadas en MATLAB-SIMULINK

\begin{tabular}{|l|l|}
\hline Capacitancias & $\begin{array}{l}\text { Estimación inicial } \\
\text { con las funciones de } \\
\text { transferencia }\end{array}$ \\
\hline $\mathrm{C} 1$ & $7.15 \mathrm{uF}$ \\
\hline $\mathrm{C} 2$ & $7.08 \mathrm{uF}$ \\
\hline $\mathrm{C} 12$ & $4.02 \mathrm{uF}$ \\
\hline
\end{tabular}

Fuente: elaboración propia

\subsection{Estimación de parámetros en MATLAB-SIMULINK}

Obtenidos todos los valores iniciales para realizar la estimación de parámetros se procede a ingresar todos los datos en SIMULINK para poder obtener los valores y curvas de voltaje simuladas. En la Figura 7 se puede observar el circuito en el entorno de trabajo de SIMULINK y en la Figura 8 se aprecia el resultado de las curvas simuladas y experimentales realizadas.

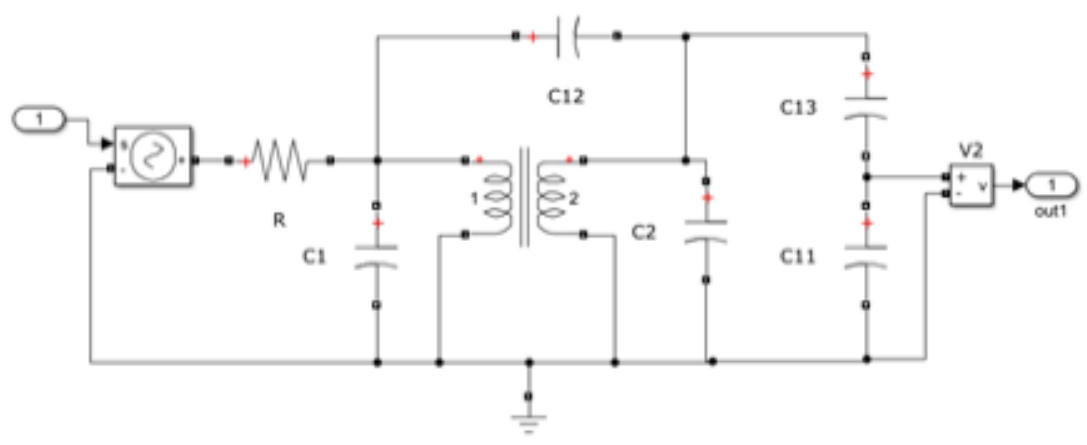

Figura 7. Modelo utilizado en la simulación de MATLAB-SIMULINK

Fuente: elaboración propia 


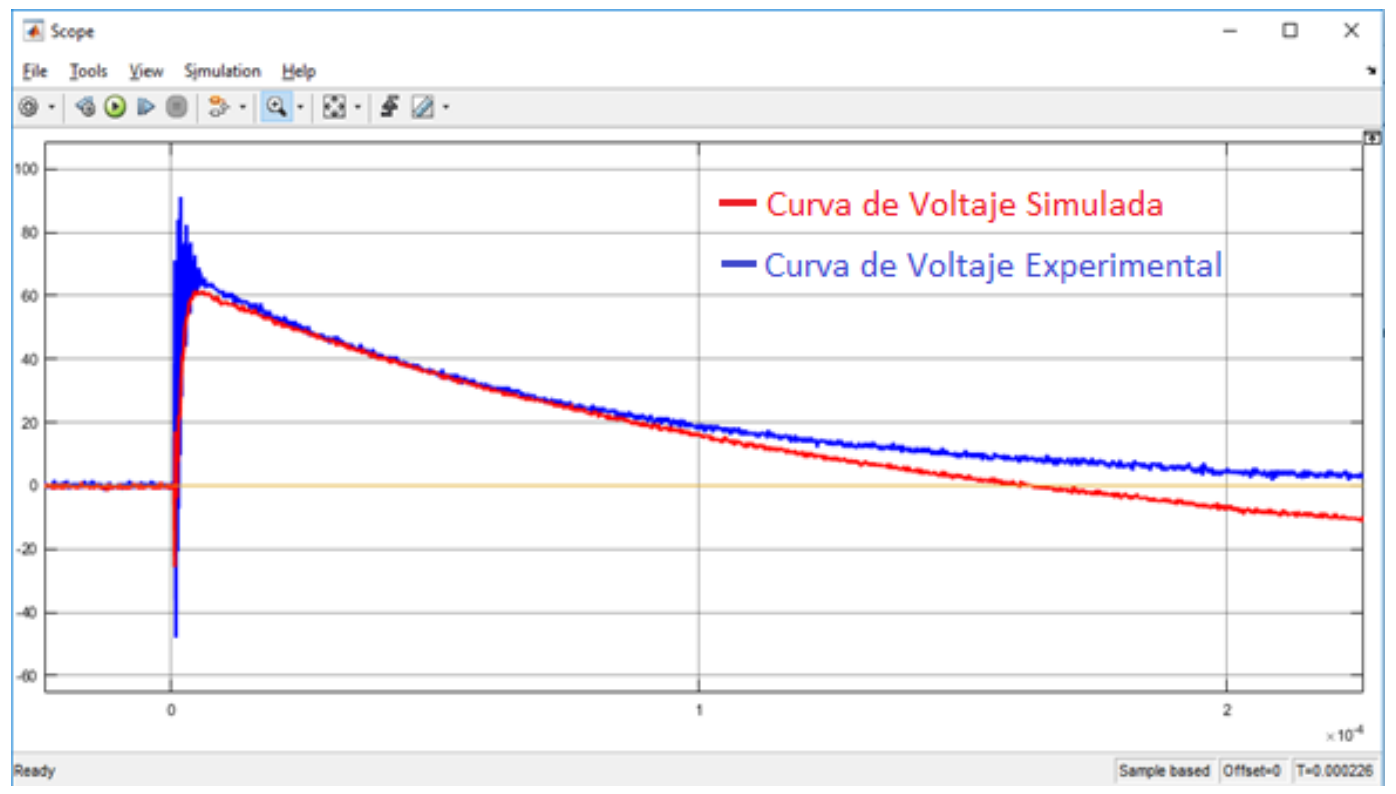

Figura 8. Curvas de voltaje obtenidas a la salida del transformador (Curva azul-forma experimental vs Curva roja-Simulación) utilizado en la simulación de MATLAB-SIMULINK

Fuente: elaboración propia

Se emplea la herramienta System Identification Toolbox para la estimación o sintonización de parámetros del modelo, esta herramienta está incluida en MATLAB-SIMULINK; se seleccionaron las curvas a comparar y los parámetros a sintonizar, en la Figura 9 se observa una captura de la interfaz del programa, el uso de esta herramienta se puede encontrar a detalle en la referencia citada (Chiguano, 2018). 


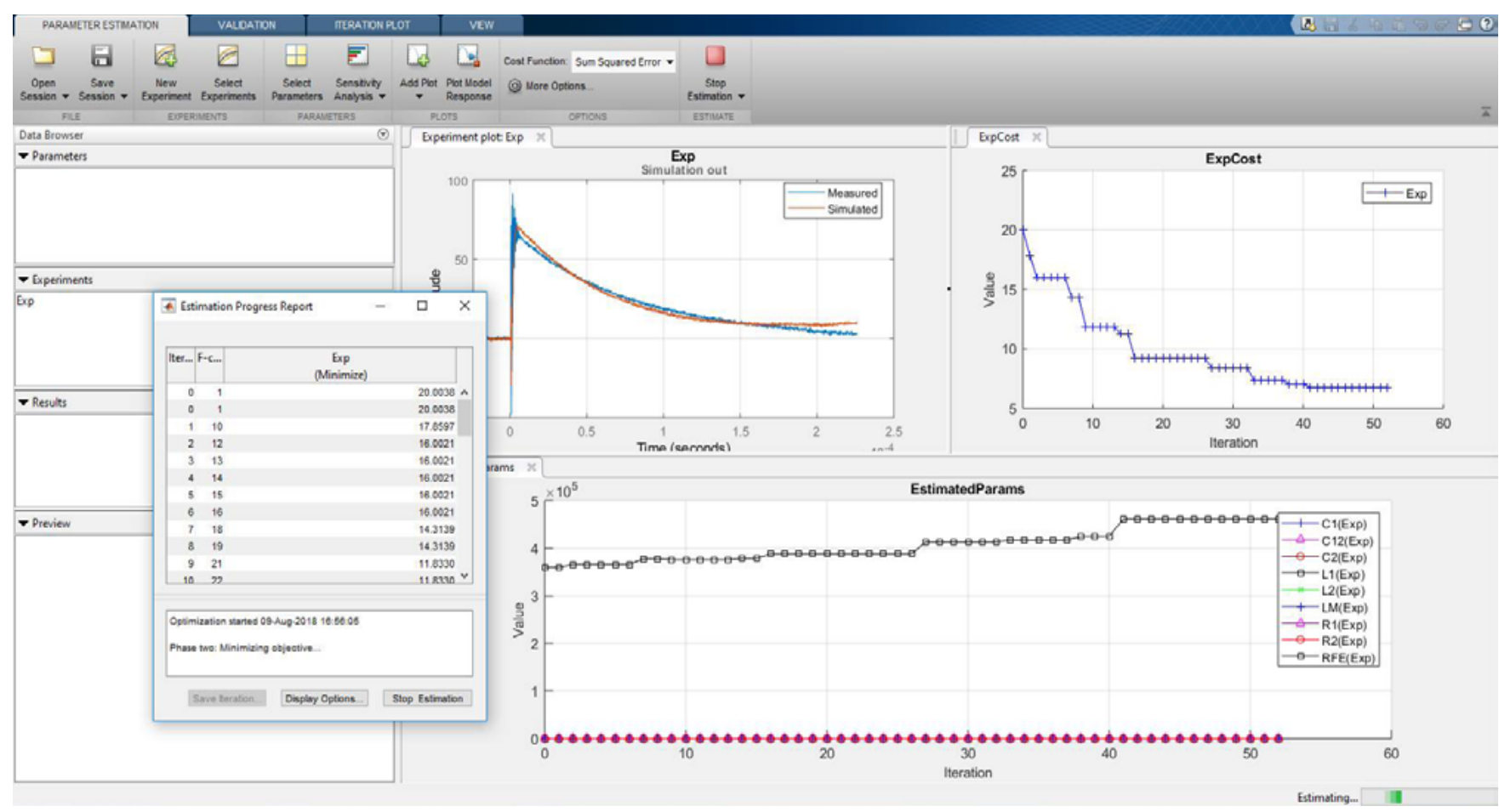

Figura 9. Estimación parámetros eléctricos transformador 50kVA en MATLAB-SIMULINK

Fuente: elaboración propia

La optimización de parámetros consiste en obtener una función objetivo o función del costo, la cual calcula una diferencia entre la respuesta simulada y la respuesta medida del transformador, esto mediante la variación de las variables de diseño del modelo que en este trabajo serían los parámetros del modelo utilizado (MathWorks, 2019).

La formulación en el proceso de optimización con la herramienta de Simulink depende del método que se desee utilizar. Los métodos de optimización no lineales minimizan la función objetivo, es decir, minimizan la diferencia entre las curvas medidas y las simuladas, variando los parámetros de su modelo.

Los métodos que se pueden utilizar son: búsqueda simple y patrones de búsqueda; con los algoritmos de Levenberg-Marquadt, punto interior, conjunto activo, región de confianza reflexiva, Programación Cuadrática Secuencial (SQP); mínimos cuadrados no lineales, gradiente descendente (Chiguano, 2018).

A continuación, en la Figura 10 se presenta un diagrama de flujo como resumen de la metodología descrita: 


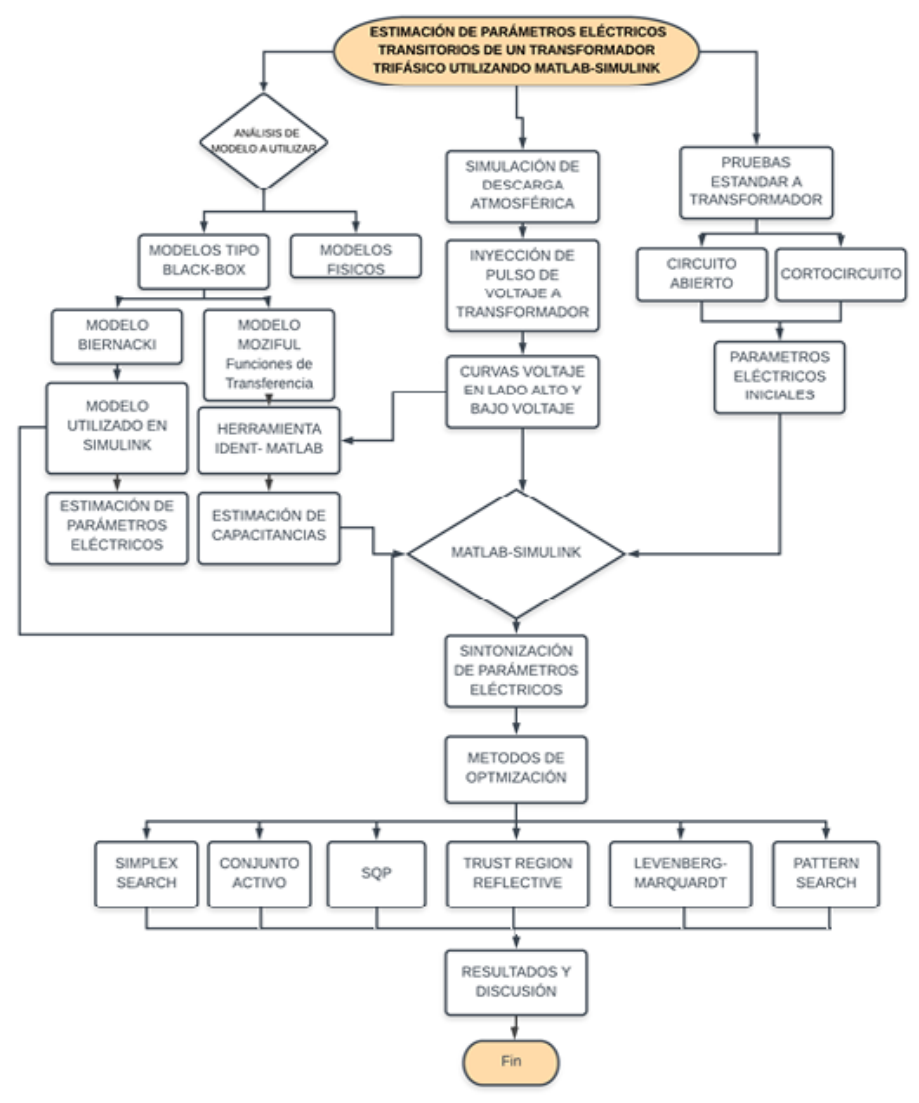

Figura 10. Diagrama de flujo de la metodología.

Fuente: elaboración propia

\section{Resultados}

Los resultados finales y valores iniciales fueron comparados mediante el cálculo del error relativo porcentual para observar la diferencia entre los valores obtenidos en simulación con los valores calculados con las pruebas estándar de cortocircuito y circuito abierto al transformador.

Una vez que la herramienta optimiza los parámetros se obtiene una curva aproximada. En la Figura 11 se presentan las curvas medida y estimada luego de la estimación de parámetros. 


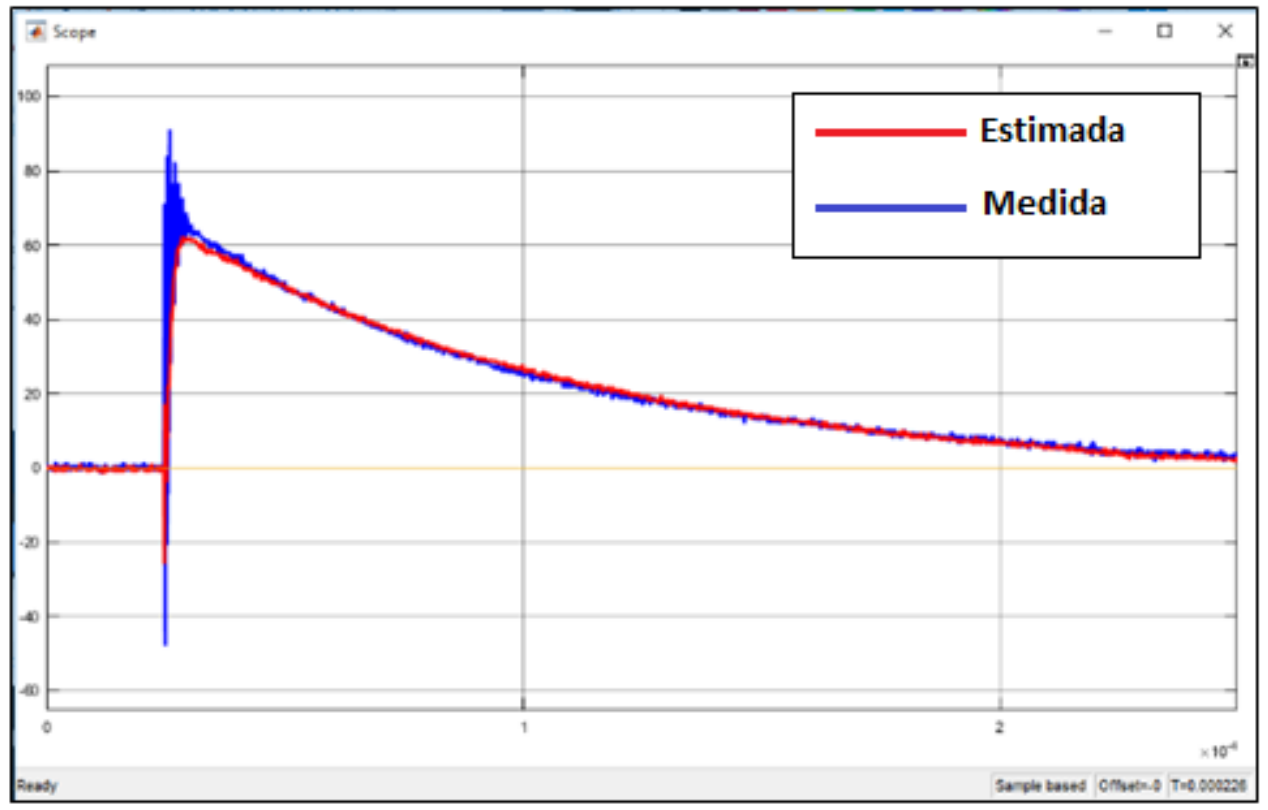

Figura 11. Curvas resultantes luego de la estimación de parámetros en MATLAB-SIMULINK

Fuente: elaboración propia

En la Tabla 4 se presentan los valores iniciales comparados con los valores estimados por MATLAB-SIMULINK, se observa que el error relativo porcentual entre los parámetros estimados y los parámetros medidos referenciales están alrededor del $20 \%$ en sus valores más elevados, estos resultados serían aceptables pues son valores pequeños, se debe tomar en cuenta también que un transformador opera continuamente en una red eléctrica, por lo que se puede presentar un deterioro no solamente en parámetros eléctricos, sino también en parámetros físicos como es el aislamiento que protege a los devanados y que inciden en el valor de las capacitancias que se presentan en el modelo.

La estimación se realizó por todos los métodos disponibles en la herramienta estimador de parámetros de MATLAB-SIMULINK, siendo el mejor método Levenberg-Marquardt pues los resultados presentan un error relativo porcentual menor con respecto a los demás métodos computacionales, por otra parte, el método con una variación más alta en el cálculo del error porcentual fue el método Simplex Search, esto se puede apreciar en la Figura 12. 
Tabla 4.

Parámetros resultantes promediados de todos los métodos de estimación

\begin{tabular}{|c|c|c|c|c|c|c|c|}
\hline \multirow{2}{*}{\multicolumn{2}{|c|}{$\begin{array}{l}\text { Parámetros } \\
\text { Cálculados } \\
\end{array}$}} & \multicolumn{2}{|c|}{$\begin{array}{l}\text { Método } \\
\text { Simplex Search }\end{array}$} & \multicolumn{2}{|c|}{$\begin{array}{l}\text { Método } \\
\text { Conjunto Activo }\end{array}$} & \multicolumn{2}{|l|}{$\begin{array}{l}\text { Método } \\
\text { SQP }\end{array}$} \\
\hline & & \multirow{2}{*}{$\begin{array}{l}\text { Estimado } \\
10.59 \Omega \\
\end{array}$} & \multirow{2}{*}{\begin{tabular}{|l|} 
Error $_{\mathrm{RP}} \%$ \\
$16.27 \%$ \\
\end{tabular}} & \multirow{2}{*}{\begin{tabular}{|l|} 
Estimado \\
$10.63 \Omega$ \\
\end{tabular}} & \multirow{2}{*}{\begin{tabular}{|l|} 
Error $_{\mathrm{RP}} \%$ \\
$16.54 \%$ \\
\end{tabular}} & \multirow{2}{*}{$\begin{array}{l}\text { Estimado } \\
9.87 \Omega \\
\end{array}$} & \multirow{2}{*}{$\begin{array}{l}\text { Error }_{\mathrm{RP}} \% \\
10.12 \% \\
\end{array}$} \\
\hline$R_{1}$ & $8.87 \Omega$ & & & & & & \\
\hline$R_{2 P}$ & $8.87 \Omega$ & $11.15 \Omega$ & $20.47 \%$ & $10.51 \Omega$ & $15.60 \%$ & $9.99 \Omega$ & $11.22 \%$ \\
\hline $\mathrm{R}_{\mathrm{FE}}$ & $360 \mathrm{k} \Omega$ & $405 \mathrm{k} \Omega$ & $11.18 \%$ & $407 \mathrm{k} \Omega$ & $11.47 \%$ & $397 \mathrm{k} \Omega$ & $9.21 \%$ \\
\hline $\mathrm{L}_{1}$ & $\begin{array}{l}34.06 \\
\mathrm{mH} \\
\end{array}$ & $34.57 \mathrm{mH}$ & $1.48 \%$ & $35.25 \mathrm{mH}$ & $3.37 \%$ & $37.08 \mathrm{mH}$ & $8.14 \%$ \\
\hline $\mathrm{L}_{2 \mathrm{P}}$ & $\begin{array}{l}34.06 \\
\mathrm{mH}\end{array}$ & $41.70 \mathrm{mH}$ & $18.32 \%$ & $33.48 \mathrm{mH}$ & $1.74 \%$ & $37.60 \mathrm{mH}$ & $9.42 \%$ \\
\hline$L_{M}$ & $90.21 \mathrm{H}$ & $95.33 \mathrm{H}$ & $5.37 \%$ & $98.38 \mathrm{H}$ & $8.30 \%$ & $95.49 \mathrm{H}$ & $5.53 \%$ \\
\hline $\mathrm{C}_{1}$ & $7.15 \mathrm{uF}$ & $7.88 \mathrm{uF}$ & $9.30 \%$ & $7.69 \mathrm{uF}$ & $7.06 \%$ & $7.79 \mathrm{uF}$ & $8.16 \%$ \\
\hline $\mathrm{C}_{2}$ & $7.08 \mathrm{uF}$ & $7.86 \mathrm{uF}$ & $9.91 \%$ & $7.55 \mathrm{uF}$ & $6.25 \%$ & $7.73 \mathrm{uF}$ & $8.42 \%$ \\
\hline \multirow[t]{2}{*}{$\mathrm{C}_{12}$} & $4.02 \mathrm{uF}$ & $4.28 \mathrm{uF}$ & $6.05 \%$ & $4.34 \mathrm{uF}$ & $7.42 \%$ & $4.20 \mathrm{uF}$ & $4.29 \%$ \\
\hline & & \multicolumn{2}{|c|}{$\begin{array}{l}\text { Método } \\
\text { Trust Region Reflective }\end{array}$} & \multicolumn{2}{|c|}{$\begin{array}{l}\text { Método } \\
\text { Levenberg-Marquardt }\end{array}$} & \multicolumn{2}{|c|}{$\begin{array}{l}\text { Método } \\
\text { Pattern Search }\end{array}$} \\
\hline \multicolumn{2}{|c|}{$\begin{array}{l}\text { Parámetros } \\
\text { Cálculados }\end{array}$} & Estimado & Error $_{\mathrm{RP}} \%$ & Estimado & Error $_{\mathrm{RP}} \%$ & Estimado & Error $_{\mathrm{RP}} \%$ \\
\hline $\mathrm{R}_{1}$ & $8.87 \Omega$ & $9.70 \Omega$ & $8.57 \%$ & $9.75 \Omega$ & $9.05 \%$ & $10.74 \Omega$ & $17.39 \%$ \\
\hline$R_{2 P}$ & $8.87 \Omega$ & $10.30 \Omega$ & $13.90 \%$ & $9.90 \Omega$ & $10.43 \%$ & $10.90 \Omega$ & $18.60 \%$ \\
\hline $\mathrm{R}_{\mathrm{FE}}$ & $360 \mathrm{k} \Omega$ & $385 \mathrm{k} \Omega$ & $6.38 \%$ & $373 \mathrm{k} \Omega$ & $3.56 \%$ & $392 \mathrm{k} \Omega$ & $8.26 \%$ \\
\hline $\mathrm{L}_{1}$ & $\begin{array}{l}34.06 \\
\mathrm{mH}\end{array}$ & $36.84 \mathrm{mH}$ & $7.54 \%$ & $36.90 \mathrm{mH}$ & $7.71 \%$ & $37.08 \mathrm{mH}$ & $8.16 \%$ \\
\hline $\mathrm{L}_{2 \mathrm{P}}$ & $\begin{array}{l}34.06 \\
\mathrm{mH} \\
\end{array}$ & $37.51 \mathrm{mH}$ & $9.21 \%$ & $34.86 \mathrm{mH}$ & $2.29 \%$ & $39.12 \mathrm{mH}$ & $12.94 \%$ \\
\hline $\mathrm{L}_{\mathrm{M}}$ & $90.21 \mathrm{H}$ & $98.30 \mathrm{H}$ & $8.23 \%$ & $93.67 \mathrm{H}$ & $3.69 \%$ & $99.79 \mathrm{H}$ & $9.60 \%$ \\
\hline $\mathrm{C}_{1}$ & $7.15 \mathrm{uF}$ & $7.46 \mathrm{uF}$ & $4.16 \%$ & $7.29 \mathrm{uF}$ & $1.98 \%$ & $7.54 \mathrm{uF}$ & $5.23 \%$ \\
\hline $\mathrm{C}_{2}$ & $7.08 \mathrm{uF}$ & $7.25 \mathrm{uF}$ & $2.33 \%$ & $7.32 \mathrm{uF}$ & $3.25 \%$ & $7.65 \mathrm{uF}$ & $7.42 \%$ \\
\hline $\mathrm{C}_{12}$ & $4.02 \mathrm{uF}$ & $4.28 \mathrm{uF}$ & $6.15 \%$ & $4.21 \mathrm{uF}$ & $4.46 \%$ & $4.23 \mathrm{uF}$ & $4.94 \%$ \\
\hline
\end{tabular}

Fuente: elaboración propia 


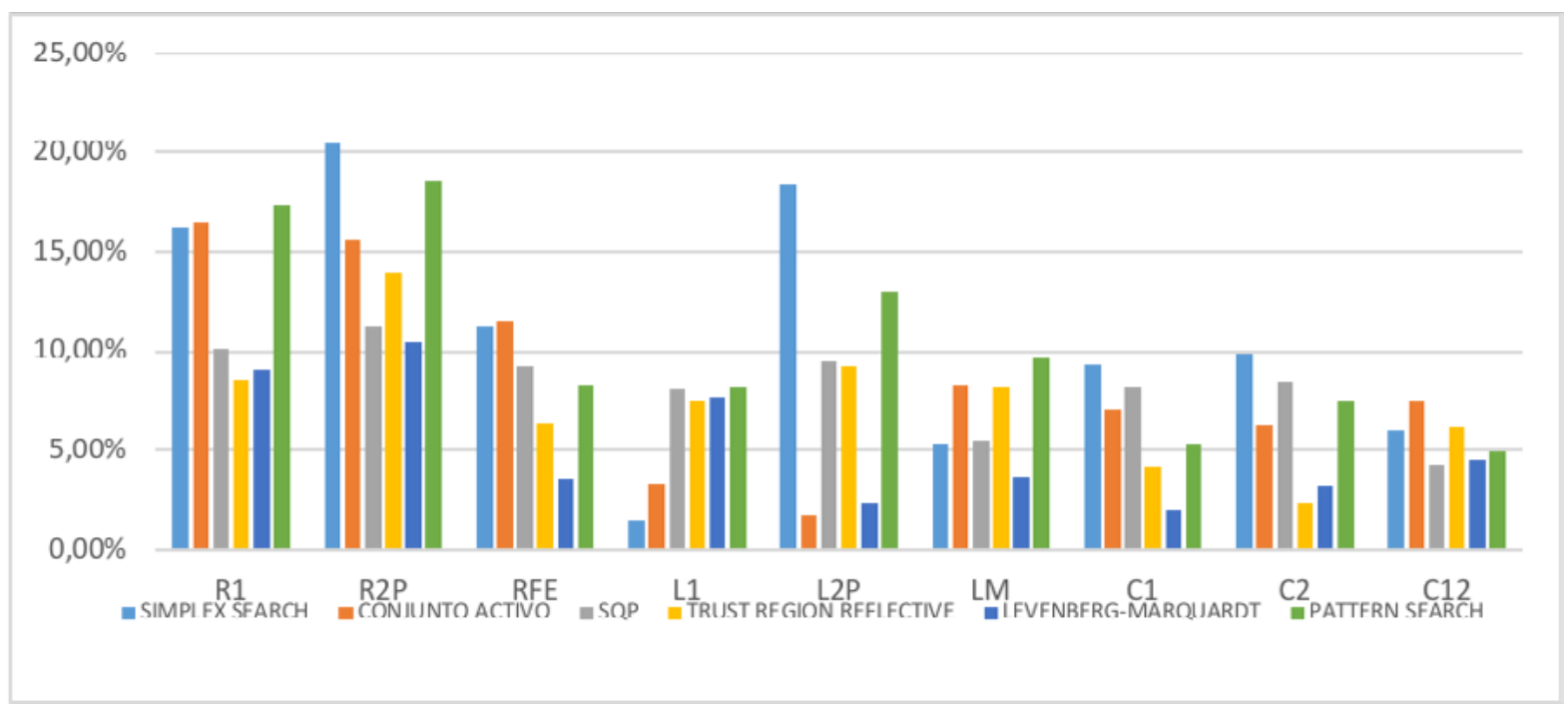

Figura 12. Error relativo porcentual entre los parámetros calculados y estimado por los diferentes métodos de estimación Fuente: elaboración propia

\section{Conclusiones}

La estimación o sintonización de parámetros mediante señales de entrada y respuesta ante eventos transitorios es una buena alternativa a cálculos tradicionales mediante pruebas estándar; sin embargo, cuando se trabaja en altas frecuencias se presentan capacitancias parásitas en el equipo, lo cual puede ser una limitante para obtener un correcto resultado, pese a esto, en el presente trabajo se cumplió con el objetivo de estimar los parámetros del modelo, teniendo una diferencia porcentual con valores máximos de alrededor del $20 \%$ como se menciona en la sección de resultados.

Las herramientas que presenta el software MATLAB-SIMULINK son de gran ayuda en la resolución de modelos matemáticos y ecuaciones diferenciales, en este estudio permitió conseguir la sintonización de los parámetros eléctricos transitorios del transformador de $50 \mathrm{kVA}$, datos que generalmente para su obtención sería necesario el uso de equipos especializados y un uso del tiempo considerable.

Los resultados con menor error porcentual fueron los obtenidos mediante el método LevenbergMarquardt como se presentaron en la Tabla 4 y en la Figura 12, por lo cual es el método recomendado para obtener los mejores resultados. 


\section{Referencias}

Abed, N.Y., Mohammed, O.A. (2010). Physics-Based High-Frequency Transformer Modeling by Finite Elements. Magnetics, IEEE Transactions on Magnetics, vol. 46, no. 8, 3249-3252. https://doi.org/10.1109/ TMAG.2010.2048017

Biernacki, J., Czarkowski, D. (2001). High frequency transformer modeling. In ISCAS 2001 - 2001 IEEE International Symposium on Circuits and Systems, The 2001 IEEE International Symposium on Circuits and Systems (Cat. No. 01CH37196), (Vol. 3, pp. 676-679). IEEE. https://doi.org/10.1109/ISCAS.2001.921401

Chiguano, B., Ramírez, J., Quilumba, F., \& Gallardo, C. (2018). Estimación de los Parámetros Eléctricos de un Generador Sincrónico basada en Mediciones de Laboratorio usando Métodos de Optimización No Lineal. Revista Técnica Energía. 15(1), 30-43. https://doi.org/10.37116/revistaenergia.v15.n1.2018.321

Galván, V., Gutiérrez, J., Ortiz, V., Sánchez, R. (2012). Modelado del transformador para eventos de alta frecuencia. Ingeniería Energética, 33(2), 122-132. http://scielo.sld.cu/scielo.php?script=sci arttext\&pi$\underline{d=S 1815-59012012000200005 \& \mid n g=e s \& t \operatorname{lng}=e s}$

Xexplore, I. (2015). C57.12.00-2015 - IEEE Standard for General Requirements for Liquid-Immersed Distribution, Power, and Regulating Transformers. IEEE https://doi.org/10.1109/IEEESTD.2016.7469278

MathWorks. (2019). How the Software Formulates Parameter Estimation as an Optimization Problem -MATLAB \& Simulink-MathWorks América Latina. https://la.mathworks.com/help/sldo/ug/optimization-problemformulation-for-parameter-estimation.html\#d117e4545

Mork, B. A., Gonzalez, F., Ishchenko, D., Stuehm, D. L., \& Mitra, J. (2007). Hybrid transformer model for transient simulation-Part I: Development and parameters. IEEE Transactions on Power Delivery, 22(1), 248-255.

Moziful Islam, S., Coates, K. M., \& Ledwich, G. (1997, October). Identification of high frequency transformer equivalent circuit using Matlab from frequency domain data. In IAS'97. Conference Record of the 1997 IEEE Industry Applications Conference Thirty-Second IAS Annual Meeting (Vol. 1, pp. 357-364). IEEE. https://ieeexplore.ieee.org/abstract/document/643049

NMX-J-169-ANCE-2004. (2004). Transformadores y Autotransformadores de Distribución y Potencia”, Métodos de Prueba.

Tibanlombo, V. (2018). Estudio de la respuesta en frecuencia mediante pruebas de impulso para la evaluación del estado del aislamiento en transformadores. EPN http://bibdigital.epn.edu.ec/handle/15000/19719 
Copyright (c) 2021 Víctor Hugo Tibanlombo Timbila, Andrés Alfredo Guevara Betancourt y Juan David Ramírez Guasgua.

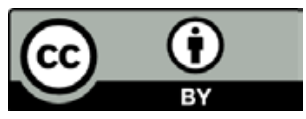

Este texto está protegido bajo una licencia internacional Creative Commons 4.0.

Usted es libre para Compartir-copiar y redistribuir el material en cualquier medio o formato - y Adaptar el documento - remezclar, transformar y crear a partir del material-para cualquier propósito, incluso para fines comerciales, siempre que cumpla las condiciones de Atribución. Usted debe dar crédito a la obra original de manera adecuada, proporcionar un enlace a la licencia, e indicar si se han realizado cambios. Puede hacerlo en cualquier forma razonable, pero no de forma tal que sugiera que tiene el apoyo del licenciante o lo recibe por el uso que hace de la obra.

Resumen de licencia - Texto completo de la licencia 